\title{
CCD Observations of Forgotten Cepheids
}

\author{
David L. DuPuy ${ }^{1}$, W. Todd Viar ${ }^{1}$ \\ Raymond H. Bloomer ${ }^{2}$, Bradley J. Ward ${ }^{2}$ \\ ${ }^{1}$ Department of Physics and Astronomy \\ Virginia Military Institute, Lexington, Virginia 24450 \\ ${ }^{2}$ Department of Physics \\ U.S. Air Force Academy, Colorado Springs, Colorado 80840
}

\begin{abstract}
CCD observations have been obtained of eight Cepheids which have few recent observations, or an uncertain period, or an uncertain finder chart. All CCD images were flat-fielded, with 4-min exposures (0.5-m telescope) or 6-min exposures (0.4-m telescope). The stars chosen for observation were CY Aqr, EV Aur, V395 Cas, V588 Cas, DW Per, HZ Per, MM Per, and SX Per.

Photometry shows clean, Cepheid light curves for six of these eight stars, with the results for MM Per shown as an example below. V588 Cas did not show a periodicity in our data set (only 9 points), and the results for EV Aur will be published separately. We obtained standard deviations of comparisonstar differences for all useable stars in our CCD images, to aid future observers in choosing suitable comparisons.
\end{abstract}

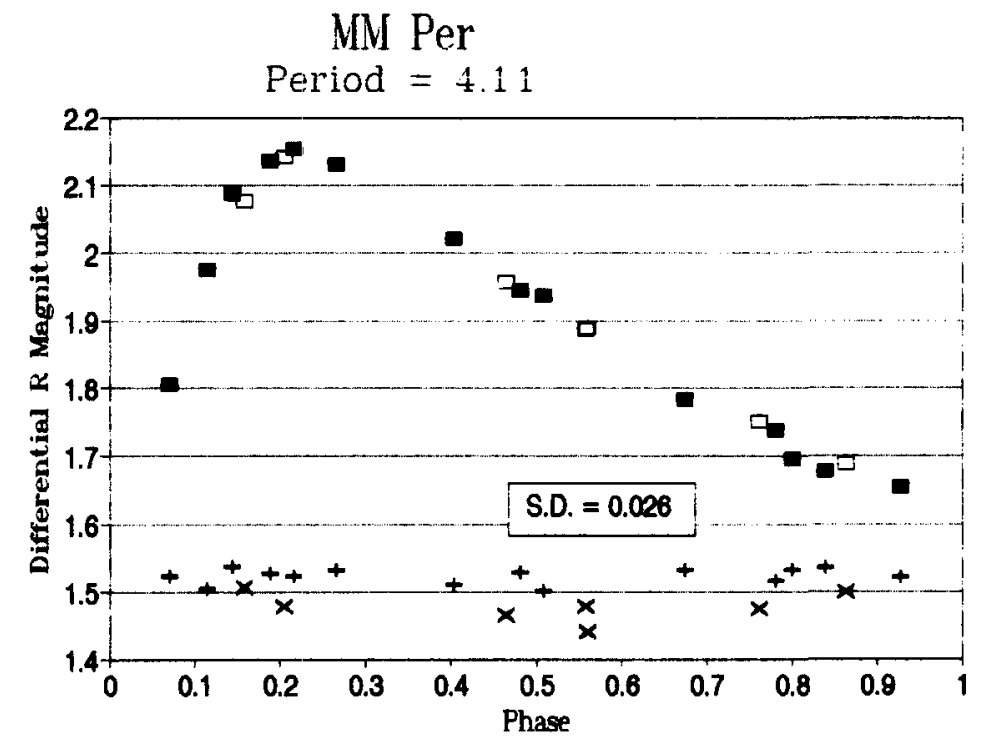

